\title{
Erratum to: Effectiveness of a Tai chi Qigong program in promoting health-related quality of life and perceived social support in chronic obstructive pulmonary disease clients
}

\author{
Aileen W. K. Chan $\cdot$ Albert Lee $\cdot$ \\ Lorna K. P. Suen · Wilson W. S. Tam
}

Published online: 15 June 2010

(C) Springer Science+Business Media B.V. 2010

Erratum to: Qual Life Res (2010) 19:653-664

DOI 10.1007/s11136-010-9632-6

The Acknowledgements section (shown below) was omitted from the original publication.

\section{Acknowledgements}

The Health and Health Services Research Fund (HHSRF), the Food and Health Bureau, and the Hong Kong SAR Government are acknowledged as the funding sources of this study.

The online version of the original article can be found under doi:10.1007/s11136-010-9632-6.

A. W. K. Chan $(\bowtie) \cdot$ L. K. P. Suen

The Nethersole School of Nursing, Faculty of Medicine,

The Chinese University of Hong Kong, Esther Lee Building,

Shatin, New Territories, Hong Kong

e-mail: aileenchan@cuhk.edu.hk

\section{A. Lee}

Department of Community and Family Medicine, Faculty

of Medicine, The Chinese University of Hong Kong, Shatin,

New Territories, Hong Kong

W. W. S. Tam

School of Public Health, The University of Hong Kong,

Pokfulam Road, Pokfulam, Hong Kong 